phys. stat. sol. (b) 127, 49 (1985)

Subject classification: 6 and $20.1 ; 22.4 .1$

Department of Physics, University of Florida, Gainesville ${ }^{1}$ )

\title{
Far-Infrared Dielectric Function of Zincblende ZnS
}

\author{
By
}

\author{
A. Memon and D. B. Tanner
}

The complex dielectric response function of zincblende $\mathrm{ZnS}$ is determined at $300 \mathrm{~K}$ by dispersive Fourier transform spectroscopy. The frequency range studied, 150 to $450 \mathrm{~cm}^{-1}$, spans the frequency of the $q=0$ transverse optic (TO) phonon mode. The frequency dependence of the anharmonic self-energy function $\Gamma(v)$ of the $q=0$ TO phonon is calculated from these data. The features observed in the spectrum of $\Gamma(v)$ are satisfactorily assigned to multi-phonon processes by comparison to theoretical lattice dynamics calculations for cubic $\mathrm{ZnS}$.

Par la methode de spectroscopie dispersive utilisant la transformee de Fourier, nous avons determine la fonction complexe de response dielectrique du $\mathrm{ZnS}$ a $300 \mathrm{~K}$. La gamme de frequences etudiees, 150 à $450 \mathrm{~cm}^{-1}$, encadre la frequence du mode phonon optique transverse a $q=0$. Les caracteristiques observees dans le spectre of $\Gamma(v)$ peuvent etre attribuees de manière satisfaisante processus multi-phonon par comparaison avec les calculs de dynamique du reseau pour la forme cubique du $\mathrm{ZnS}$.

\section{Introduction}

The effects of anharmonic lattice dynamics on the properties of crystals have been studied by many investigators [1 to 3]. According to Cowley [2] anharmonic interactions in alkali halide crystals lead to considerable structure in the self-energies of the transverse optic (TO) and longitudinal optic (LO) phonons at frequencies near that of the LO mode. Similar results have been obtained for the case of III-V and II-VI compound semiconductors in studies which employed dispersive Fourier transform spectroscopy (DFTS) to determine the far infrared dielectric response functions $[4,5]$.

In this paper we present a DFIS measurement of the far infrared complex dielectric function and anharmonic self-energy function $\Gamma(v)$ of zincblende $\mathrm{ZnS}$. We show that structure in $\Gamma(v)$ can be related to multiphonon processes in the lattice dynamics of the crystal, making DFTS a useful method to determine precise phonon frequencies at the zone center and major symmetry points.

\section{Experimental Techniques}

An asymmetric Michelson interferometer [6] was used to measure the far infrared reflection amplitude and phase of $\mathrm{ZnS}$. In the DFTS [2] technique used in these measurements the sample replaces the mirror at the end of the fixed arm of the Michelson interferometer. The sample simultaneously reduces the amplitude and shifts the phase of the light traveling in the fixed arm relative to the case when a metallic mirror is at the end of the fixed arm. Thus the spectrum produced by the interferometer has an amplitude and phase which are respectively the amplitude reflectivity, $r$, and phase shift upon reflection, $\varphi$, of the sample. These quantities

1) Gainesville, Florida 32611, USA.

4 physica (b) $128 / 1$ 
yield the complex refractive index, $N$,

$$
N=\frac{1+r \mathrm{e}^{i \varphi}}{1-r \mathrm{e}^{i \varphi}}
$$

which in turn is related to the complex dielectric function $\varepsilon$ by

$$
\varepsilon=N^{2} \text {. }
$$

Our measurements were made at $300 \mathrm{~K}$ on a $\mathrm{ZnS}$ single crystal $2.5 \times 3.7 \mathrm{~cm}^{2}$ in size, considerably larger than the $1.8 \mathrm{~cm}$ diameter of the far infrared beam striking the sample. The frequency range where useful results were obtained was 150 to $450 \mathrm{~cm}^{-1}$ at an instrumental resolution of $4 \mathrm{~cm}^{-1}$. The average standard deviation of the data give errors of \pm 0.01 in the amplitude spectrum and of \pm 0.03 radian in the phase spectrum. Because the anharmonic self-energie function is more sensitive to the measured phase than the amplitude, the phase errors below 250 and above $450 \mathrm{~cm}^{-1}$ are large enough to give large errors in the measured anharmonic self-energy; hence, we report the anharmonic self-energy function only for 250 to $450 \mathrm{~cm}^{-1}$. The error bars shown in this spectrum are determined from an average over several runs.

\section{Results and Discussion}

The experimentally measured amplitude reflection coefficient and phase reflection spectra for $\mathrm{ZnS}$ in the frequency range 150 to $450 \mathrm{~cm}^{-1}$ are presented in Fig. 1. The real and imaginary parts of the complex dielectric response function obtained using the above data are shown in Fig. 2. The zone center longitudinal optic phonon (LO) and transverse optic phonon (TO) frequencies are indicated in the figure. The LO frequency was determined using Drude's method which consists of finding the frequency at which the real part of dielectric function, $\varepsilon_{3}(v)$, goes through zero. The TO frequency was determined from the position of the maximum in the imaginary part, $\varepsilon_{2}(v)$, of the dielectric response function. The values of $\mathrm{TO}$ and $\mathrm{LO}$ so obtained are listed and compared with previous results [ 7 to 9$]$ in Table 1 . It can be seen that our values are in excellent agreement with those obtained by other techniques.

In polar crystals the lattice absorption bands arise from the direct interaction of infrared radiation with the crystal lattice phonons. The strongest of these interaction

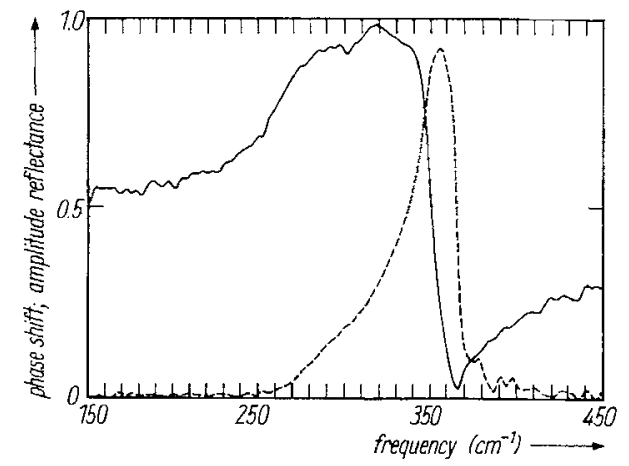

Fig. 1

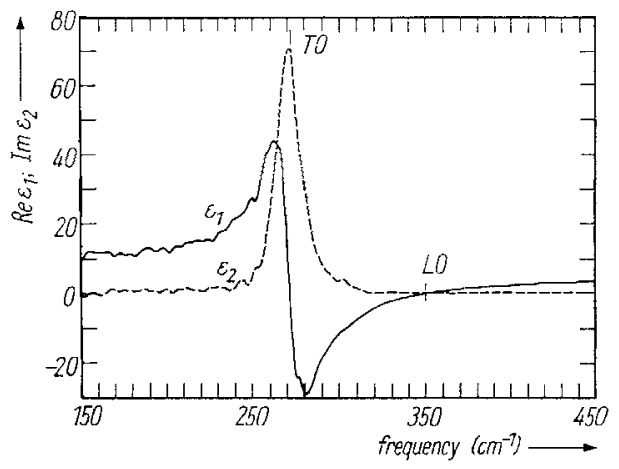

Fig. 2

Fig. 1. Far infrared amplitude reflectance (full curve) and phase shift $(\varphi-\pi)$ upon reflection (dashed curve) from $\mathrm{ZnS}$

Fig. 2. Real (full curve) and imaginary (dashed curve) far-infrared dielectric function of ZnS 
Table 1

Observed long wavelength optical phonons for cubic $\mathrm{ZnS}\left(\mathrm{cm}^{-1}\right)$

\begin{tabular}{lll}
\hline technique & TO & LO \\
\hline this work (DFTS) & 273 & 350 \\
reflection [7] & 274 & 350 \\
measurements & & \\
neutron scattering [8] & $\mathbf{2 7 4} \pm \mathbf{3}$ & $\mathbf{3 5 0} \pm \mathbf{3}$ \\
Raman scattering [9] & $\mathbf{2 7 1}$ & $\mathbf{3 5 2}$ \\
\hline
\end{tabular}

is between a photon, and a single long wavelength optical phonon (the $q=0$ TO phonon) which gives rise to the main resonance bands. Besides this band there are other weaker bands which are more important from our point of view because they determine the details of the anharmonic self-energy function. These bands arise from the interaction of an incident photon with a pair of phonons. Two types of mechanism have been proposed as responsible for this sort of coupling: anharmonic forces [10] or second order electric moment [11]. These mechanisms are described in detail by Kleinman [10] and Lax [11] respectively.

To calculate the full frequency dependence of the imaginary part of the anharmonic self-energy, $\Gamma(v)$, we follow the procedure described in a previous paper [12], assuming that the structure in anharmonic self-energy functions $\Gamma(v)$ arises primarily due to anharmonic forces. We are able to calculate $\Gamma(\nu)$, using our measured values of $\varepsilon_{1}(v), \varepsilon_{2}(v)$, and $\nu_{\text {To }}$ and taking the values of $\varepsilon_{1}(0)$ and $\varepsilon(\infty)$ from [13]. These quantities are related by

$$
\Gamma(v)=\frac{v_{\mathrm{To}}[\varepsilon(0)-\varepsilon(\infty)] \varepsilon_{2}(v)}{2\left\{\left[\varepsilon_{1}(v)-\varepsilon(\infty)\right]^{2}+\varepsilon_{2}^{2}(v)\right\}}
$$

The anharmonic self-energy function $\Gamma(v)$ so calculated is shown in Fig. 3.

We now propose the assignments for the observed features in the spectrum of $\Gamma(v)$ in terms of two phonon decay processes. The critical point frequencies at $\mathrm{X}, \mathrm{L}$, and $W$ have been calculated on the basis of a simple lattice dynamical model by Talwar and Agrawal [9]. Within the experimental resolution most of the features observed and marked in the spectrum of $\Gamma(v)$ shown in Fig. 3, agree quite well with the frequencies in this calculation. The major discrepancies occur at the features numbered 3, 4, and 5 . These discrepancies can be removed by careful choice of the longitudinal acoustic frequencies $\mathrm{LA}(\mathrm{L})$ and $\mathrm{LA}(\mathrm{X})$. Our selection of $\mathrm{LA}(\mathrm{L})=195 \mathrm{~cm}^{-1}$ is $6 \mathrm{~cm}^{-1}$ higher than that calculated by Talwar and Agrawal. This value for LA(L)

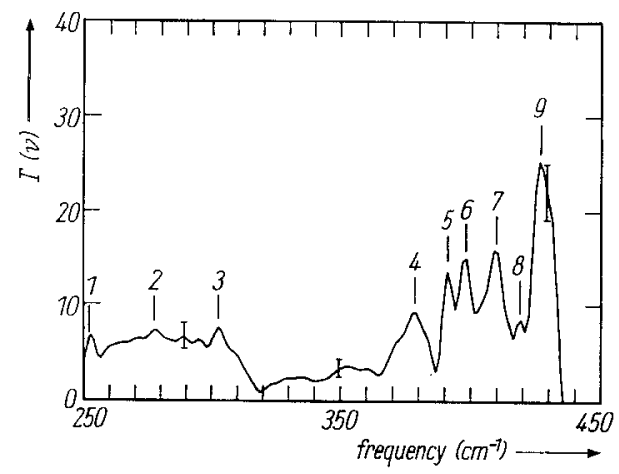

Fig. 3. Phonon self-energy function $\Gamma(v)$ for $\mathrm{ZnS}$. Marked features are listed in Table 2 
Table 2

Assignments of features in Fig. 3 in terms of two phonon summation process

\begin{tabular}{|c|c|c|c|}
\hline features & assignments & $\begin{array}{l}\text { expected } \\
\text { freq. }\left(\mathrm{cm}^{-1}\right)\end{array}$ & $\begin{array}{l}\text { observed } \\
\text { freq. }\left(\mathrm{cm}^{-1}\right)\end{array}$ \\
\hline 1 & $W_{1}+W_{2}$ & 252 & 252 \\
\hline 2 & $\mathbf{L A}(\mathbf{L})+\mathbf{T A}(\mathbf{L})$ & 273 & 272 \\
\hline 3 & $\mathrm{LA}(\mathrm{X})+\mathrm{TA}(\mathrm{X})$ & 301 & 302 \\
\hline 4 & $\mathrm{TO}(\mathrm{L})+\mathrm{TA}(\mathrm{L})$ & 373 & 377 \\
\hline 5 & $2 \mathrm{LA}(\mathrm{L})$ & 390 & 391 \\
\hline 6 & $\mathrm{TO}(\mathrm{X})+\mathrm{TA}(\mathrm{X})$ & 402 & 399 \\
\hline 7 & $\mathrm{LO}(\mathrm{X})+\mathrm{TA}(\mathrm{X})$ & 413 & 411 \\
\hline 8 & $\mathrm{LO}(\mathrm{L})+\mathrm{TA}(\mathrm{L})$ & 422 & 420 \\
\hline 9 & $W_{1}+W_{6}$ & 428 & 427 \\
\hline
\end{tabular}

exactly matches neutron scattering data [8] and also accounts well for the prominent peaks at features 3 and 4, marked in Fig. 3, which cannot otherwise be explained. Similarly the calculated value for $\operatorname{LA}(X)\left(203 \mathrm{~cm}^{-1}\right)$ is lower than neutron scattering measurements. We chose $\mathrm{LA}(\mathrm{X})=211 \mathrm{~cm}^{-1}$, close to the neutron scattering values, and are thus able to explain the feature (5). The feature numbered (9) was assigned using the selected frequencies of [9] at the $W$ symmetry point. Thus, our measurements as well as neutron scattering data suggest that the theoretical lattice dynamical model calculation of Talwar and Agrawal does not account very well for the longitudinal acoustic frequency at major symmetry points, $\mathrm{X}$ and $\mathrm{L}$.

The complete list of expected and observed features is given in Table II, from which one can see that the observed features agree quite well with the calculated frequencies. Thus the technique of DFTS if carefully performed can provide improved values for the phonon frequencies at the zone center and major symmetry points in the Brillouin zone.

\section{Acknowledgments}

A. Memon gratefully acknowledges the Council for International Exchange of Scholars for their award of Senior Fulbright Postdoctoral Scholarship and wishes to thank the Department of Physics, University of Florida for their hospitality. We are thankful to Mrs. Sheri Hill for typing this manuscript.

\section{References}

[1] M. BorN and K. Huang, Dynamical Theory of the Crystal Lattice, Oxford University Press 1954.

[2] R. A. Cowley, Adv. Phys. 12, 421 (1963).

[3] J. I. BerG and E. E. BeLL, Phys. Rev. B 4, 3572 (1971).

[4] A. Memon and T. J. Parker, Proc. Soc. Photo-Opt. Instrum. Engng. 289, 20 (1981).

[5] T. J. Parker, J. R. Btrch, and C. L. Mok, Solid State Commun. 35, 581 (1980).

[6] E. E. Russell and E. E. BeLl, Infrared Phys. 6, 75 (1966).

[7] M. Balkansisi, M. Nusimovicr, and R. Le Toulec, J. Phys. 25, 305 (1964).

[8] N. Vagelatos, D. Wehe, and J. S. King, J. chem. Phys. 60, 9, 3613 (1974).

[9] D. N. Talwar and Bal K. Agrawal, phys. stat. sol. (b) 64, 71 (1974).

[10] D. A. Kreinman, Phys. Rev. 118, 118 (1960).

[11] M. Lax and E. Burstern, Phys. Rev. 97, 39 (1955).

[12] A. Memon and D. B. Tanner, Infrared and Millimeter Waves, 5, 10091984.

[13] C. Hass and J. P. Mathieu, J. Phys. Radium 15, 492 (1954). 